\title{
Extensión Universitbaria: altra proyección socio-educativa y un aporte significativo para el desarrollo del país
}

C. Copyright 2021. Universidad Nacional Autónoma de Nicaragua, Managua (UNAN-Managua) Todos los derechos reservados

\section{University Extension: high socio-educational projection and a significant contribution to the development of the country}

Zenayda Aurora Suárez ${ }^{1}$

https://orcid.org/0000-0002-9582-797

Fecha de recibido: 07/05/2021

\section{Resumen}

La Extensión Universitaria, ofrece una importante estrategia para el desarrollo del país desde diferentes ámbitos, los principales protagonistas son los estudiantes, en ellos recae una de las funciones de extensión universitaria de gran impacto social, como son: las prácticas de formación, profesionalización y pasantías, a través de las cuales, se potencializa la articulación entre la formación y la investigación, sin duda alguna, esta función favorece la integralidad de los estudiantes y de los demás miembros de la comunidad universitaria.

Formar individuos reflexivos y comprometidos es una responsabilidad compartida tanto de la universidad como la sociedad y las familias; el vínculo sociedad -

\author{
Miriam Patricia Téllez Marín ${ }^{2}$ \\ https://orcid.org/0000-0002-3737-9734
}

Fecha de dictaminado: 31/05/202

universidad permite además el logro de competencias de los estudiantes en su campo de profesionalización, asimismo la inserción al campo laboral.

La institución de educación superior, tiene un fuerte compromiso social en cuanto a extender su accionar hacia los diferentes estratos de la sociedad; sin duda, permite fortalecer las relaciones de cooperación e intercambio de conocimiento, cuya estrategia es de prioridad para la institución educativa.

La gestión de la universidad, se fortalece cada vez que las estrategias desarrolladas en la práctica, tienen el reconocimiento social del impacto observado de manera satisfactoria. La misión de la universidad, incorpora los elementos claves para seguir su marcha al desarrollo y competitividad (Castillo, E, 2020) 


\section{Palabras claves}

Proyección social, compromiso, cooperación, integración e intercambio.

\section{Summary}

The university extension offers an important strategy for the development of the country from different areas, the main protagonists are the students who perform one of the functions of university extension of great social impact, such as: training practices, professionalization and internships, Through these, the articulation between training and research is strengthened; Without a doubt, this function favors the integrality of the students and the other members of the university community.

Forming thoughtful and committed individuals is a shared responsibility of both the university and society and families; the society-university link also allows students to achieve competencies in their field of professionalization, as well as insertion into the labor field.

The institution of higher education has a strong social commitment in terms of extending its actions to the different strata of society; undoubtedly, it allows to strengthen the relations of cooperation and exchange of knowledge, whose strategy is a priority for the educational institution.

The management of the university is strengthened each time that the strategies developed in practice have the social recognition of the impact observed in a satisfactory way.

The mission of the university incorporates the key elements to continue its progress towards development and competitiveness (Castillo, E 2020)

\section{Keywords}

Social projection, commitment, cooperation, integration and exchange.

\section{Introducción}

El concepto de extensión universitaria ha tomado fuerza en los últimos años, vinculando los conceptos de innovación, creatividad y emprendimiento, los cuales se han popularizado en la oferta de productos y servicios, vistos como una forma de negocio que genera ingresos. No obstante, desde la perspectiva de vinculación de la sociedad con la universidad, el concepto de extensión, traslada a la universidad fuera de las aulas de clases, conectándose directamente con la población, desde los diferentes entornos, mediante la práctica profesional. Su aporte se ve reflejado en el acompañamiento a los programas de gobierno, fortaleciendo la calidad de los servicios ofrecidos a la población.

La universidad, tiene una importante función en la formación de jóvenes integrales y creativos, que generen desarrollo personal, familiar y social; estas competencias, posicionan a la universidad, en un alto nivel de responsabilidad para el desarrollo socioeconómico del país (Castillo, E., Valenzuela, Silva \& Romero, J., 2020).

Un alto compromiso de esta entidad, se ve reflejado en la motivación de toda la comunidad universitaria, produciendo, creando e innovando este salto importante, que resalta en las diferentes esferas sociales, gubernamentales y no gubernamental. Promocionando el talento de los jóvenes, con mentes brillantes que aportan a la competitividad interna y externa.

\section{Rol de la Universidad}

La universidad como tal, no es una empresa que diseña un producto y lo lleva al mercado para su comercio; sin embargo, el desarrollo del pensamiento lógico en los estudiantes potencializa el servicio creativo, innovador y sostenible, al integrarse en diferentes tareas interinstitucionales; por ejemplo, los que están en el área de la agronomía se enfocan en cooperación con MARENA, MAG y Alcaldías, apoyando desde asesoría técnica, pero también en fuerza física cuando se involucran de manera directa en el cuido del medio ambiente: cuencas hidrográficas, reforestación, sanidad animal, tecnificación de suelos para la agricultura, entre otros. Por otro lado, los estudiantes de las áreas de la salud, se integran directamente en todos los programas de salud preventiva, asistencia directa y rehabilitación; de igual forma existe acompañamiento por docentes que aportan sus conocimientos en la facilitación de los aprendizajes que garantizando el cumplimiento de una diversidad de actividades.

El empoderamiento de los estudiantes, en cuanto a las Tecnologías de la información y la comunicación 
(TIC), les permite trabajar la creatividad e innovación en solución de problemas sociales, a través, de sistemas web o aplicaciones móviles muy útiles, trasladándolos al desarrollo económico y social de la población. Además, en el año 2020, se demandó el uso de plataformas educativas, que ayudaron a la población a dar continuidad al proceso de enseñanzaaprendizaje desde casa. (Gutiérrez, H. (2020). Hoy día, los estudiantes de la FAREM Chontales, participan como protagonistas de proyectos innovadores en las diferentes ferias promovidas por el estado, en función del desarrollo comunitario a través de la economía creativa.

\section{¿Cómo valora la sociedad, la inserción de la universidad en la comunidad?}

La población en general, observa de manera positiva, todas las formas en que la Universidad se vincula socialmente; existe confianza y aceptación en todas las actividades que promueve la universidad fuera del aula, tales como: campañas, ferias, capacitaciones, acompañamiento y/o adiestramiento entre otros.

Estas acciones, están generando desarrollo del talento humano, en creatividad y emprendimiento, a través de los cales se está observando la sostenibilidad del país (Chacón. O. A, 2018)

\section{¿Cómo se vincula la extensión universitaria con las instituciones gubernamentales?}

El talento humano mantiene vínculo directo, con la formación de los estudiantes, generando servicios a la población, a través de las prácticas de asistencia comunitaria —en cualquier contexto hace una fuerte producción de servicios-, al aplicarla la creatividad, la innovación, para el fortalecimiento de los programas sociales mediante el aporte científico y tecnológico, esto garantiza el acompañante técnico a la población emprendedora, más allá de poner en práctica los conocimientos teóricos, contribuyen el desarrollo del país.

Algunas disciplinas, se ubican de manera literal en primera línea de acción, en materia de colaboración con las instituciones del estado, tal como se apreció en el contexto de la Pandemia de COVID-19, estudiantes y maestros formaron parte activa de las campañas de educación para la prevención y control de esta, al igual que el involucramiento en actividades de prevención ante las multiamenazas por desastres naturales, donde la universidad estuvo en primera línea, apoyando actividades de cooperación solidaria, ante la catástrofe de los dos huracanes que azotaron Nicaragua (Betanco, J. A., Reyes, E. A., Rodríguez, Pérez, M 2017)

De manera, que la extensión universitaria, es una función clave que constituye una forma de articulación entre la formación y la investigación, así mismo, favorece la formación integral de los estudiantes y de los demás miembros de la comunidad universitaria, fortalece la formación de individuos, como sujetos activos reflexivos y comprometidos con la sociedad; de tal manera, que el concepto de extensión Universitaria se convierte en una responsabilidad social de la institución, para ampliar su quehacer, llegando hasta las familias, la comunidad y la sociedad; otra importante actividad se vislumbra desde su misión en el fortalecimiento de las relaciones de cooperación, acompañamiento e intercambio de conocimientos, posesionándose en las estrategias de mayor prioridad dentro del campo de acción de la institución (Castillo E, 2020).

¿Cómo se vinculan las diferentes facultades en el desarrollo de la extensión universitaria con las instituciones públicas?

La Universidad nunca ha estado ausente, su relación de cooperación en diferentes actividades de carácter social, — de acompañante-, han tenido una valoración positiva por diferentes entidades; aunque las tareas no se acoplaban al enfoque directo y complejo que lo amerita la extensión universitaria, a continuación, se hace una amplia descripción de como algunas facultades tanto nacionales como regionales se han destacado en el área de la extensión social.

La "Facultad Regional Multidisciplinaria de Chontales (FAREM-Chontales)" desde el área de la salud pública, marcó su inicio en el año 2008 en la segunda etapa de la revolución cuando se reivindicó el derecho a una salud de calidad inclusiva y gratuita encaminada a una salud preventiva llevando servicios de salud a las diferentes comunidades, en la casa a casa, en las unidades de salud tanto primarios como secundarios. Fue entonces que la facultad dio un salto importante en el trabajo comunitario con un alto nivel de aceptación por la población y autoridades sanitarias, al momento en que los estudiantes se integraron en la contención de la epidemia de la INFLUENZA HIN1 en una campaña de grandes magnitudes siguiendo la 
estrategia de educación para la salud, desde el casa a casa, aplicación masiva de vacunas, voluntariado en la atención a los pacientes afectados por la epidemia que fueron ingresados a las unidades de salud, en este proceso se incluyeron los docentes para acompañar a los estudiantes en esta misión, lo que vino a aliviar un poco la carga de trabajo para los trabajadores sanitarios.

Pero la Facultad no detuvo su camino en este ámbito, el 2015, también marcó su camino en integración al trabajo colaborativo en las diferentes unidades de salud, respecto a la contención de la epidemia de Chikungunya, integrándose de la misma forma a la campaña de educación para la salud.

Las carreras de salud de esta Facultad, han creado vínculo directo con la entidad pública Ministerio de Salud (MINSA), como ente rector del sector salud, en cumplimiento con lo establecido en la Constitución Política de la República de Nicaragua, facultado por la Ley General de Salud, desde su Modelo de Salud Familiar y Comunitario (MOSAFC), constituye una ruta para lograr la equidad en el sector, reto que obliga a garantizar el acceso a los servicios de salud y reducir las brechas de atención a los grupos más excluidos socialmente. Desde este Modelo la UNANFAREM Chontales aporta a la comunidad mediante esta integración con el ministerio de salud, para responder a las necesidades y expectativas de la población, a fin de que obtengan una atención de salud integral con calidad, calidez y respeto, donde se garantiza el derecho a la salud, pilar fundamental de las Políticas del Gobierno de Reconciliación y Unidad Nacional, caracterizada por los principios de gratuidad, universalidad, solidaridad.

Los estudiantes, desde las prácticas de profesionalización en las carreras de enfermería y medicina, se integran a este Modelo, enfocado en la atención en salud a la comunidad, donde se privilegia a cada familia nicaragüense, principalmente las más vulnerables. Asimismo, se aborda a las personas en todos los ciclos de vida como un proceso continuo, con momentos interrelacionados de promoción y prevención de su salud, atención al daño y rehabilitación con un enfoque preventivo amplio y con acciones dirigidas al control del medio ambiente. Este proceso de interrelación e integración permite el contacto directo con el individuos, la familia y la comunidad mediante una plena participación, con un espíritu de autorresponsabilidad y autodeterminación, llevando lo más cerca posible la atención de salud al lugar donde residen y trabajan las personas, constituyéndose en primer elemento de un proceso permanente de asistencia sanitaria, función central del desarrollo social y económico global de la comunidad.

Esta etapa importante en la formación de los estudiantes de las carreas de salud, permite el fortaleciendo de redes articuladas para brindar los servicios integralmente (OMS, 2019)

En el 2011, la Facultad Multidisciplinaria de Chontales, desarrolló vínculos de colaboración con la universidad de Pablo de Olavide de España, que ha venido formando alianza de colaboración en proyectos sociales; de modo que la Universidad los ejecuta en las comunidades más vulnerables, como escuelas públicas y unidades de salud. Además, se realizan campañas de cuido y preservación del medio ambiente.

En el año 2020, se alcanza una alta participación en diferentes actividades, coordinados con los ministerios gubernamentales, sin embargo, los eventos coyunturales llevaron a ubicar a la UNAN Managua y sus Facultades Regionales en un alto nivel de resolución, consolidándose el importante papel que juega la universidad desde la extensión social. Al presentarse una epidemia mortal, que necesito movilización de administrativos, estudiantes y docentes de las diferentes carreras, en apoyo a la campaña de prevención y control de medidas de higiene que disminuyeran los casos de alto riesgo en la ciudad; además, la ayuda a los afectados por dos huracanes de grandes magnitudes en la costa atlántica del país, requirió de voluntaria participación de esta entidad, en el proceso de evaluación de los daños y acompañamiento a las víctimas.

\section{Material y método}

En este documento, se describen las diferentes experiencias en participación social como actividades extensionistas, según lo definen las fuentes bibliográficas consultadas. Denotando cada actividad enriquecedora en la formación integral, dejando huellas en humanismo, servicio y transmisión de conocimientos.

El desarrollo metodológico, se realiza desde el seguimiento y control a la planificación anual de la facultad, como principal indicador del compromiso social. Aplicando un nivel de profundidad exploratorio 
que permita recopilación de información cualitativa de satisfacción y reconocimiento de la población. Se definen preguntas directrices como salida a la investigación.

\begin{tabular}{|l|c|l|}
\hline \multicolumn{1}{|c|}{ Cuestión } & \multicolumn{1}{|c|}{ Intabla 1. Directrices } \\
\hline $\begin{array}{l}\text { Desempeño de la universidad en la } \\
\text { sociedad }\end{array}$ & $\begin{array}{l}\text { ¿Cómo valora la sociedad, la } \\
\text { inserción de la universidad en la } \\
\text { comunidad? }\end{array}$ & $\begin{array}{l}\text { Incidir de forma positiva en } \\
\text { la población, insertando a la } \\
\text { comunidad universitaria en } \\
\text { la solución de problemáticas } \\
\text { presentadas. }\end{array}$ \\
\hline $\begin{array}{l}\text { Vinculación universidad - gobierno } \\
\text { y su aporte en la extensión social. }\end{array}$ & $\begin{array}{l}\text { ¿Cómo se vincula la extensión } \\
\text { universitaria con las instituciones } \\
\text { gubernamentales? }\end{array}$ & $\begin{array}{l}\text { Participar de forma activa en } \\
\text { actividades sociales convocadas } \\
\text { por instituciones del gobierno. }\end{array}$ \\
\hline $\begin{array}{l}\text { Firmas de convenios que faciliten } \\
\text { integración de la universidad en las } \\
\text { instituciones públicas. }\end{array}$ & $\begin{array}{l}\text { ¿Cómo se vinculan las diferentes } \\
\text { facultades en el desarrollo de la } \\
\text { extensión universitaria con las } \\
\text { instituciones públicas? }\end{array}$ & $\begin{array}{l}\text { Establecer convenios de } \\
\text { colaboración con instituciones } \\
\text { públicas en pro del bienestar social. }\end{array}$ \\
\hline
\end{tabular}

Fuente: Elaboración propia, adaptado de Mengo (2009)

La investigación documental se realiza a través de los pasos de la tabla siguientes:

La investigación documental se realiza a través de los pasos de la babla siguientes:

\begin{tabular}{|l|l|l|}
\hline 1.Formulación del problema & $\begin{array}{l}\text { Selección y delimitación del tema e } \\
\text { interrogantes a resolver }\end{array}$ & $\begin{array}{l}\text { Fase 1: Selección del tema o } \\
\text { problema }\end{array}$ \\
\hline 2. Ejecución de la investigación & Acopio de información, fichajes & $\begin{array}{l}\text { PFase 2: Sistematización de la } \\
\text { información }\end{array}$ \\
\hline $\begin{array}{l}\text { 3. Formulación y presentación de la } \\
\text { información }\end{array}$ & $\begin{array}{l}\text { Redacción de la información, } \\
\text { discusión, reflexión, referencias, } \\
\text { conclusiones, recomendaciones. }\end{array}$ & $\begin{array}{l}\text { Fase 3: Desarrollo del proceso, } \\
\text { análisis, interpretación de la } \\
\text { información, redacción de } \\
\text { apartado crítico, estructuración del } \\
\text { informe. }\end{array}$ \\
\hline
\end{tabular}

\section{Análisis y discusión de los resultados}

La población de Chontales, considera la participación de la universidad de manera positiva, a través de invitaciones a diferentes actividades sociales promovidas por instituciones responsables, que acreditan la transferencia de conocimientos que esta aporta, generando siempre excelentes resultados, útiles para el desarrollo económico, cultural, tecnológico y comunitario de la región. (UNAN Managua - FAREM Chontales, 2020)

La extensión universitaria, permanece en contacto directo desde la interacción técnica, científica e investigativa con todas las instituciones generadoras de servicio público, como: Alcaldía, MINSA, INTA, MEFCCA, MINED, INTUR entre otras. Desde el ámbito social, se logra un contacto directo con la población 
vulnerable, por medio de la atención y prevención de problemas comunitarios que demandan acción compartida, ejemplo: campañas de prevención de enfermedades emergentes y remergentes del entorno, campaña de uso correcto de las tecnologías, redes sociales, posibles adicciones y enfermedades visuales que pueden generarse.

La universidad, hace un trabajo interinstitucional de roles compartidos, con la finalidad de lograr el desarrollo local, interviniendo en problemas sociales enfocándose en la extensión critica desde las prácticas, pasantías, actividades deportivas, culturales, acompañamientos económicos y políticos.

\section{Conclusiones}

La Extensión Universitaria es una ventana de oportunidades para toda la comunidad universitaria, todas las carreras tienen fuertes aspectos de desarrollo de la extensión universitaria que están aportando al desarrollo económico y social del país, sin embargo, se considera como una de las formas de mayor interacción, las prácticas de formación, donde los estudiantes y maestros tienen un contacto directo con la población y sus problemas más sentidos, en el momento de la empatía ante las demandas más sentidas por cada estrato social, vista desde diferentes contextos.

\section{Referencias bibliográficas}

- Betanco, J. A., Reyes, E. A., Rodríguez, Pérez, $M$ (2017). Experiencias vinculantes de la universidad-empresa estado como estrategia clave para el crecimiento de la facultad regional multidisciplinaria Estelí de UNAN Managua

- Castillo, E (2020) definición y formas de hacer extensión universitaria. UNAN Managua. Nicaragua

- Castillo, E., Valenzuela, Silva \& Romero, J. (2020). Antecedentes y tipología de la extensión universitaria. Managua: UNAN Managua

- Chacón. O. A (2018). La enseñanza de la innovación para el desarrollo sostenible. Recuperadode:https://www.revistasnicaragua. net.ni/index.php/multiensayos/article/ view/4949/4958. 05 de febrero de 2021
- Gutiérrez H., Escobar, A. (2020). Experiencia de la UNAN-Managua en el aseguramiento de la calidad educativa en los contextos de pandemia. Política de Buen Gobierno, la resiliencia socioeducativa y la continuidad educativa en tiempos de la COVID-19. Recuperado: http://www.cnu.edu.ni/ foro-la-educacion-superior-en-nicaragua-2020-yel-impacto-de-la-covid-19/

- López-Noguero, F., Morón-Marchena, J. A y Gallardo-López. J.A (2020). Educación para la salud y desarrollo comunitario. Investigaciones para la mejora social en Nicaragua. Recuperado de: https:// octaedro.com/wp-contentuploads/2020/09/16189. pdf el 10 de feb del 2021.

- OMS (2019). intercambio experiencias y buenas prácticas de atención, promoción y participación ciudadana que implementan los diferentes Ministerios de Salud: foro. Nicaragua.

- UNAN Managua - FAREM-Chontales. (23 de 03 de 2020). Estudiantes y maestros de UNAN FAREM Chontales apoyando al MINSA en campana preventiva con el covid-19. Obtenido de https:// cutt.ly/obYzj5N 